\title{
Gaining insights into pulmonary hypertension in respiratory diseases
}

\author{
Joan Albert Barberà ${ }^{1,2}$ and Isabel Blanco ${ }^{1,2}$ \\ Affiliations: 'Dept of Pulmonary Medicine, Hospital Clínic and August Pi i Sunyer Biomedical Research \\ Institute (IDIBAPS), University of Barcelona, Barcelona, Spain. ${ }^{2}$ Biomedical Research Networking Center in
} Respiratory Diseases, Madrid, Spain.

Correspondence: Joan A. Barberà, Servei de Pneumologia, Hospital Clínic, Villarroel, 170, 08036 Barcelona, Spain. E-mail: jbarberåclinic.ub.es

○ @ERSpublications

Pulmonary hypertension associated with respiratory disease is a difficult to manage condition with significant burden http://ow.ly/S1COQ

Pulmonary hypertension (PH) associated with respiratory diseases, group 3 in the current classification [1], is one of the most frequent presentations of $\mathrm{PH}$. Chronic obstructive pulmonary disease (COPD) and idiopathic pulmonary fibrosis (IPF) are the two most common respiratory disorders associated with $\mathrm{PH}$. In these conditions the presence of $\mathrm{PH}$ is associated with shorter survival $[2,3]$ and worse clinical course [4]. Yet, much is still unknown about group $3 \mathrm{PH}$ regarding its prevalence in the whole disease spectrum, noninvasive markers that might facilitate its recognition and, most importantly, how to treat it.

The majority of studies assessing prevalence of $\mathrm{PH}$ in chronic respiratory diseases have been conducted in patients with advanced disease [5,6]. Little is known about moderate disease stages. Recent studies report a prevalence of $\mathrm{PH}$ among COPD patients in Global Initiative for Chronic Obstructive Lung Disease (GOLD) stages 2 and 3 of $7 \%$ and 25\%, respectively [7, 8], and among 8-15\% of patients with IPF at their diagnostic evaluation [3,9]. Regarding treatment of $\mathrm{PH}$, only a few trials have assessed the effects of targeted therapy in patients with chronic respiratory diseases, most of them with negative results [10-13]. For this reason current guidelines do not recommend such treatment until more evidence on its efficacy is available $[1,6]$. Two articles in this issue of the European Respiratory Journal provide further insights into group $3 \mathrm{PH}[14,15]$, particularly regarding its prevalence and response to treatment.

RAGHU et al. [14] report the characteristics and outcomes of patients with IPF and PH that participated in the ARTEMIS-IPF trial, a randomised, placebo-controlled study to evaluate the effectiveness and safety of ambrisentan in IPF. The trial was terminated prematurely because ambrisentan-treated patients were at increased risk for disease progression and respiratory hospitalisations [16]. The ARTEMIS-IPF study enrolled 494 patients with mild-to-moderate IPF and all patients underwent right heart catheterisation (RHC) at baseline. At the time of study termination, 117 patients had undergone a second RHC after 48 weeks, allowing the investigators to document prevalence and progression of $\mathrm{PH}$ in a large cohort of patients with mild-to-moderate IPF. Their results show that precapillary PH was present in $14 \%$ of patients. The reported prevalence concurs with that seen in patients with IPF at their initial evaluation $[3,9]$ and contrasts with that observed in patients with advanced IPF candidates for lung transplantation, where it is 2-3 times higher [17]. Prevalence of PH in mild-to-moderate IPF is also similar to that observed in GOLD stage 2 and 3 COPD $[7,8]$. PH was of mild severity in the majority of patients and only $4 \%$ had severe $\mathrm{PH}[6] .4 \%$ of patients developed $\mathrm{PH}$ at the follow-up RHC, with an estimated progression of pulmonary artery pressure (PAP) of $+0.17 \mathrm{mmHg}$ per month. This contrasts with advanced IPF, where the progression of PAP is much faster, $+3.8 \mathrm{mmHg}$ per month [18]. The study by RaGHu et al. [14] enlarges our knowledge of the epidemiology of $\mathrm{PH}$ in the clinical spectrum of IPF by showing that $\mathrm{PH}$ is quite prevalent in mild-to-moderate disease, although it is of mild severity and progresses slowly.

Received: Aug 042015 | Accepted after revision: Aug 062015

Conflict of interest: Disclosures can be found alongside the online version of this article at erj.ersjournals.com

Copyright @ERS 2015 
In the second article, BREWIS et al. [15] report the experience of a PH reference centre on the characteristics and response to treatment of patients with severe $\mathrm{PH}$ and coexistent respiratory disease, which were compared with idiopathic pulmonary arterial hypertension (PAH). Quite surprisingly, during the 14-year evaluation period, there were more incident cases with concomitant respiratory disease than patients with idiopathic PAH. Both groups were treated similarly. However, patients with respiratory disease had worse survival. The study reflects real life management of $\mathrm{PH}$ and illustrates the burden and clinical impact of $\mathrm{PH}$ associated with respiratory disorders. Sometimes, in patients with mild ventilatory impairment and severe $\mathrm{PH}$, the distinction between group 3 and group $1 \mathrm{PH}$ is elusive and represents a diagnostic dilemma [6]. In the series reported by BREWIS et al. [15], patients with concomitant lung diseases were older, more frequently male and smokers, and had mild ventilatory impairment, severe hypoxaemia, and markedly reduced diffusing capacity of the lung for carbon monoxide, although with a lower haemodynamic impairment as compared with idiopathic PAH. This suggests that patients with $\mathrm{PH}$ presenting this phenotype should undergo a high-resolution computed tomography scan to rule out concomitant parenchymal lung disease.

Taken together both studies tell us that $\mathrm{PH}$ is a frequent complication of chronic respiratory disease, even in patients with mild-to-moderate ventilatory impairment. Patients with associated respiratory disease represent a considerable group among patients with $\mathrm{PH}$, which is difficult to manage and has very poor outcome [19]. Despite the absence of supportive evidence, patients with $\mathrm{PH}$ and concomitant respiratory disease often receive targeted PAH therapy [20, 21]. In the study by BREWIs et al. [15], almost all patients with $\mathrm{PH}$ and lung disease were treated with $\mathrm{PAH}$ drugs, although they did not show improvement in either functional class or 6-min walking distance, and only NT-pro-brain natriuretic peptide improved significantly. In contrast, patients with idiopathic PAH showed significant improvements in all three assessments after treatment.

In the study by RAGHU et al. [14], the effect of ambrisentan was assessed in a randomised controlled trial. Treatment with ambrisentan was not associated with any haemodynamic benefit, even in the subgroup of patients with severe PH. By contrast, similar to the general study [16], ambrisentan-treated patients experienced a higher rate of disease progression events than placebo-treated patients.

In summary, both studies show, once more, that PAH-targeted therapy provided no benefit to patients with chronic respiratory diseases who had mild or severe $\mathrm{PH}$, and that in the case of ambrisentan it was detrimental in IPF.

Does this mean we must forget treating PH in patients with respiratory diseases using the currently available drugs? Perhaps, but we think that before doing so we should reconsider the end-points used to assess efficacy of $\mathrm{PH}$ treatment, as they might be inappropriate for patients with concomitant respiratory disease. The majority of studies conducted in patients with PH associated with COPD or IPF have failed to demonstrate significant improvement of exercise tolerance as the primary end-point [10-13]. Despite the fact that exercise tolerance is a clinically meaningful end-point in both PAH and respiratory disease, the determinants of its limitation are different. Whereas in PAH the main limiting factor is cardiac output [22], in patients with chronic respiratory disease ventilatory limitation and worsening of gas exchange are the major determinants of exercise tolerance $[23,24]$. Accordingly, any improvement in cardiac output with targeted therapy will translate into increased exercise capacity in $\mathrm{PAH}$, whereas in patients with chronic respiratory disease this may not occur because ventilatory and gas exchange constraints remain unchanged. Therefore, we should consider alternative end-points to assess the efficacy of $\mathrm{PH}$ treatment in parenchymal lung disease.

End-points of clinical trials are being reconsidered in PAH, because improvement in exercise tolerance is a poor predictor of long-term outcome $[25,26]$. Recent trials in PAH use composite measures that reflect clinical worsening as primary end-points with an event-driven design [27]. Presumably, this design could also be appropriate for patients with respiratory disease-associated PH. In fact, RAGHU et al. [14] used this design in their study and showed a detrimental effect of ambrisentan on disease progression in IPF. In patients with chronic lung diseases $\mathrm{PH}$, despite of being of mild severity, has been consistently associated with mortality [2, 3] and morbidity, in particular with exacerbation episodes in COPD [4, 28]. Exacerbation episodes requiring hospitalisation are associated with increased mortality in COPD [29]. Similarly, hospitalisation due to clinical worsening is associated with poor survival in PAH [30]. Recent trials demonstrate that targeted therapy significantly reduces hospitalisations in PAH [27, 31]. Although the mechanisms explaining the association between PH and COPD exacerbations are unclear [4, 28], it is conceivable that hospitalisations due to clinical worsening might provide a potential end-point in $\mathrm{PH}$ associated with respiratory disease [32].

An additional issue to consider for future clinical trials in group $3 \mathrm{PH}$ is the selection of the appropriate patient population. So far, the majority of trials have been conducted in patients with mild-to-moderate $\mathrm{PH}$ with negative results [33]. The fifth World Symposium on Pulmonary Hypertension recommended targeting patients with severe $\mathrm{PH}$ and mild or moderate parenchymal lung disease, as they appear to be 
those who might eventually benefit from a drug acting on the pulmonary vessels [6]. Although in the study by BREwis et al. [15] there were no differences between patients with milder or more severe parenchymal impairment, the lack of a control group precludes establishing definite conclusions in this respect.

In summary, the studies by RAGHU et al. [14] and BREWIS et al. [15] highlight the significant burden of PH associated with respiratory diseases and reveal the difficulties in its management. Unfortunately, both studies do not provide good news on the therapeutic approach to this condition. On the contrary, they corroborate the poor response to targeted PAH therapy and alert about the potential harmful effects in IPF. However, $\mathrm{PH}$ associated with respiratory disease is highly prevalent, patients often seek our attention, and the prognosis of this condition is extremely poor. Regrettably, we are still lacking large, multicentre controlled trials in this group of patients, as the majority of studies have been investigator-driven initiatives, conducted in a limited number of cases. We urgently need robust trials in which the objectives and the design are adapted to the specific characteristics of these patients [34]. This is the only way we will find out which is the most appropriate treatment for $\mathrm{PH}$ associated with respiratory disease. It will be difficult to undertake this type of study considering the concomitant morbidity, but given the burden of this severe condition it should be attempted. For the time being, it is better do no harm. As the study by Raghu et al. [14] shows, the use of $\mathrm{PAH}$ medications may not be innocuous and may produce detrimental effects in some respiratory diseases.

\section{References}

1 Galiè N, Humbert M, Vachiery J-L, et al. 2015 ESC/ERS Guidelines for the diagnosis and treatment of pulmonary hypertension. Eur Respir J 2015; 46: 903-975.

2 Andersen $\mathrm{KH}$, Iversen $\mathrm{M}$, Kjaergaard J, et al. Prevalence, predictors, and survival in pulmonary hypertension related to end-stage chronic obstructive pulmonary disease. J Heart Lung Transplant 2012; 31: 373-380.

3 Kimura $\mathrm{M}$, Taniguchi $\mathrm{H}$, Kondoh $\mathrm{Y}$, et al. Pulmonary hypertension as a prognostic indicator at the initial evaluation in idiopathic pulmonary fibrosis. Respiration 2013; 85: 456-463.

4 Wells JM, Washko GR, Han MK, et al. Pulmonary arterial enlargement and acute exacerbations of COPD. $N$ Engl J Med 2012; 367: 913-921.

5 Oliveira RK, Pereira CA, Ramos RP, et al. A haemodynamic study of pulmonary hypertension in chronic hypersensitivity pneumonitis. Eur Respir J 2014; 44: 415-424.

6 Seeger W, Adir Y, Barbera JA, et al. Pulmonary hypertension in chronic lung diseases. J Am Coll Cardiol 2013; 62: Suppl., D109-D116.

7 Hilde JM, Skjørten I, Hansteen V, et al. Haemodynamic responses to exercise in patients with COPD. Eur Respir J 2013; 41: 1031-1041.

8 Portillo K, Torralba Y, Blanco I, et al. Pulmonary hemodynamic profile in chronic obstructive pulmonary disease. Int J Chron Obstruct Pulmon Dis 2015; 10: 1313-1320.

9 Hamada K, Nagai S, Tanaka S, et al. Significance of pulmonary arterial pressure and diffusion capacity of the lung as prognosticator in patients with idiopathic pulmonary fibrosis. Chest 2007; 131: 650-656.

10 Blanco I, Santos S, Gea J, et al. Sildenafil to improve respiratory rehabilitation outcomes in COPD: a controlled trial. Eur Respir J 2013; 42: 982-992.

11 Corte TJ, Keir GJ, Dimopoulos K, et al. Bosentan in pulmonary hypertension associated with fibrotic idiopathic interstitial pneumonia. Am J Respir Crit Care Med 2014; 190: 208-217.

12 Goudie AR, Lipworth BJ, Hopkinson PJ, et al. Tadalafil in patients with chronic obstructive pulmonary disease: a randomised, double-blind, parallel-group, placebo-controlled trial. Lancet Respir Med 2014; 2: 293-300.

13 Hoeper MM, Halank M, Wilkens $\mathrm{H}$, et al. Riociguat for interstitial lung disease and pulmonary hypertension: a pilot trial. Eur Respir J 2013; 41: 853-860.

14 Raghu G, Nathan SD, Behr J, et al. Pulmonary hypertension in idiopathic pulmonary fibrosis with mild-to-moderate restriction. Eur Respir J 2015; 46: 1370-1377.

15 Brewis MJ, Church AC, Johnson MK, et al. Severe pulmonary hypertension in lung disease: phenotypes and response to treatment. Eur Respir J 2015; 46: 1378-1389.

16 Raghu G, Behr J, Brown KK, et al. Treatment of idiopathic pulmonary fibrosis with ambrisentan: a parallel, randomized trial. Ann Intern Med 2013; 158: 641-649.

17 Shorr AF, Wainright JL, Cors CS, et al. Pulmonary hypertension in patients with pulmonary fibrosis awaiting lung transplant. Eur Respir J 2007; 30: 715-721.

18 Nathan SD, Shlobin OA, Ahmad S, et al. Serial development of pulmonary hypertension in patients with idiopathic pulmonary fibrosis. Respiration 2008; 76: 288-294.

19 Chaouat A, Naeije R, Weitzenblum E. Pulmonary hypertension in COPD. Eur Respir J 2008; 32: 1371-1385.

20 Trammell AW, Pugh ME, Newman JH, et al. Use of pulmonary arterial hypertension-approved therapy in the treatment of non-group 1 pulmonary hypertension at US referral centers. Pulm Circ 2015; 5: 356-363.

21 Deaño RC, Glassner-Kolmin C, Rubenfire M, et al. Referral of patients with pulmonary hypertension diagnoses to tertiary pulmonary hypertension centers: the multicenter RePHerral study. JAMA Intern Med 2013; 173: 887-893.

22 Sun XG, Hansen JE, Oudiz RJ, et al. Exercise pathophysiology in patients with primary pulmonary hypertension. Circulation 2001; 104: 429-435.

23 Troosters T, Vilaro J, Rabinovich R, et al. Physiological responses to the 6-min walk test in patients with chronic obstructive pulmonary disease. Eur Respir J 2002; 20: 564-569.

24 Blanco I, Villaquiran C, Valera JL, et al. Peak oxygen uptake during the six-minute walk test in diffuse interstitial lung disease and pulmonary hypertension. Arch Bronconeumol 2010; 46: 122-128.

25 Savarese G, Paolillo S, Costanzo P, et al. Do changes of 6-minute walk distance predict clinical events in patients with pulmonary arterial hypertension? A meta-analysis of 22 randomized trials. J Am Coll Cardiol 2012; 60: 1192-1201.

26 Gomberg-Maitland M, Bull TM, Saggar R, et al. New trial designs and potential therapies for pulmonary artery hypertension. J Am Coll Cardiol 2013; 62: Suppl., D82-D91. 
27 Pulido T, Adzerikho I, Channick RN, et al. Macitentan and morbidity and mortality in pulmonary arterial hypertension. N Engl J Med 2013; 369: 809-818.

28 Kessler R, Faller M, Fourgaut G, et al. Predictive factors of hospitalization for acute exacerbation in a series of 64 patients with chronic obstructive pulmonary disease. Am J Respir Crit Care Med 1999; 159: 158-164.

29 Soler-Cataluña JJ, Martínez-García MA, Román Sánchez P, et al. Severe acute exacerbations and mortality in patients with chronic obstructive pulmonary disease. Thorax 2005; 60: 925-931.

30 Frost AE, Badesch DB, Miller DP, et al. Evaluation of the predictive value of a clinical worsening definition using 2-year outcomes in patients with pulmonary arterial hypertension: a REVEAL Registry analysis. Chest 2013; 144: 1521-1529.

31 Channick RN, Delcroix M, Ghofrani HA, et al. Effect of macitentan on hospitalizations: results from the SERAPHIN trial. JACC Heart Fail 2015; 3: 1-8.

32 Nathan SD, King CS. Treatment of pulmonary hypertension in idiopathic pulmonary fibrosis: shortfall in efficacy or trial design? Drug Des Devel Ther 2014; 8: 875-885.

33 Barberà JA, Blanco I. Management of pulmonary hypertension in patients with chronic lung disease. Curr Hypertens Rep 2015; 17: 62 .

34 Cottin V. Treatment of pulmonary hypertension in interstitial lung disease: do not throw out the baby with the bath water. Eur Respir J 2013; 41: 781-783. 